\title{
удк: $616.147 .22-007.64$
}

\section{ВАРИКОЦЕЛЭКТОМИЯ У ПОДРОСТКОВ - КОГО И КОГДА НАДО ОПЕРИРОВАТЬ}

\section{Сизонов В.В. ${ }^{1}$, Макаров А.Г. ${ }^{1}$, Коган М.И. ${ }^{2}$}

${ }^{1}$ Детское уроандрологическое отделение

ГБУ РО «Областная детская больница», г.Ростов-на-Дону

${ }^{2}$ Кафедра урологии и репродуктивного здоровья человека с курсом детской урологии-андрологии ГБОУ ВПО РостГМУ Минздрава России, г.Ростов-на-Дону

Эл.почта: vsizonov@mail.ru, lexxrgmu@yandex.ru,dept_kogan_mail.ru

Статья содержит литературный обзор посвященный проблеме варикоиеле, тактики ведения пациентов с данной патологией и определения показаний к оперативному лечению.

Ключевые слова: варикочеле, варикоцелэктомия

\section{VARICOCELE FOR ADOLESCENT - WHOM AND WHEN TO OPERATE}

\section{Sizonov V.V. ${ }^{1}$, Makarov A.G. ${ }^{1}$, Kogan M.I. ${ }^{2}$}

${ }^{1}$ Children's Uro-Andrology Department of Regional Children's Hospital, Rostov-on-Don

${ }^{2}$ Department of Urology and Human Reproductive Health with a Course of Pediatric Urology-Andrology Rostov State Medical University, Rostov-on-Don

The article contains a literature review is devoted to the problem of varicocele, tactics of patients with this pathology and the determination of indications for surgical treatment.

Key words: varicocele, varicocelectomy

B арикозное расширение вен семенного канатика - самое частое поражение органов репродуктивной системы у подростков. В возрасте до 10 лет заболеваемость составляет менее $1 \%$, с началом полового созревания она возрастает выше 15\% [1, 2]. В основу стратегии профилактической варикоцелэктомии у детей и подростков положены данные Всемирной организации здравоохранения о выявлении у 20-40\% мужчин, обратившихся за помощью в связи с инфертильностью, варикоцеле [3] и результатах исследований, свидетельствующих о снижении качества спермы у больных с варикоцеле по сравнению со здоровыми мужчинами [4].
Важным обстоятельством, побуждающим к хирургической активности в подростковом возрасте, являются данные о наивысшей степени восстановления тестикулярного объема после варикоцелэктомии, выполненной до 14 лет [5].

С другой стороны нет прямых научных доказательств негативного влияния варикоцеле на репродуктивную функцию. Кроме того, существуют исследования, свидетельствующие о временном характере улучшения показателей спермограммы у пациентов после варикоцелэктомии $[6,7]$, что чрезвычайно актуально при решении вопроса о сроках лечения детей и подростков, учи- 
тывая отдаленность периода реализации репродуктивной функции.

Бельгийские коллеги опубликовали исследование [8], в котором изучили целесообразность выполнения варикоцелэктомии у детей и подростков, исходя из вероятности отцовства в будущем. Приведенные ими данные демонстрируют, что в группе больных, которых вели консервативно удалось стать отцами 85\% больных, в то время как среди оперированных отцовство было достигнуто в 78\%. Авторы делают вывод об отсутствии положительного влияния на вероятность отцовства в будущем активной хирургической тактики при лечении варикоцеле у детей и подростков.

Острота поднятой проблемы иллюстрируется публикацией в том же номере двух статей содержащих комментарии к работе бельгийских коллег. Kolon [9] указывая на слабые точки доказательной базы бельгийского исследования, высказывает уверенность в необходимости дальнейших поисков, направленных на выявление группы пациентов нуждающихся в раннем хирургическом вмешательстве. В комментарии редактора высказывается принципиальное несогласие с полученными выводами о нецелесообразности хирургии у подростков и высказывается уверенность в необходимости разработки совершенных критериев отбора тех 20\% подростков с варикоцеле, которые будут иметь проблемы с репродуктивной функцией в будущем.
В современном диагностическом арсенале, используемом при обследовании подростка с бессимптомным варикоцеле, важнейшей составляющей считается изучение тестикулярного объема. Первые публикации [10-15] посвященные зависимости между наличием тестикулярной атрофии у подростков и будущим нарушением репродуктивной функции, сводились к констатации факта без указания значения степени уменьшения объема тестикул. При этом исследование параметров спермы не выполнялось, а критерием эффективности варикоцелэктомии считали увеличение объема яичка на стороне варикоцеле после хирургического лечения.

На следующем этапе изучения гипотрофии яичка при варикоцеле были разработаны критерии оценки степени гипотрофии основанные на изучении абсолютных значений объема тестикул $[16,17]$ и параметров спермограмы. Kass et al. опубликовали результаты исследования взаимосвязи между степенью гипотрофии яичка и ухудшением параметров спермограммы, которые позволили им утверждать, что снижение объема яичка ипсилатерального варикоцеле на 2,0 $\mathrm{cm}^{3}$ и более является уровнем, который можно использовать как критерий отбора пациентов, нуждающихся в варикоцелэктомии.

Использование абсолютных значений обладает существенным недостатком, так как при оценке степени атрофии не учитывается возрастные и ин- 
дивидуальные особенности конституции. Понятно, что дефицит тестикулярного объема 2,0 $\mathrm{cm}^{3}$ у подростков в начале пубертатного периода отражает более выраженный атрофический процесс, чем у подростков, завершивших половое развитие. Описанные очевидные недостатки абсолютных значений привели к поиску критериев, использующих относительные показатели отражающие степень тестикулярной гипотрофии. Наибольшее распространение получила формула расчета, использующая при расчете объемы правого и левого яичка:

$\frac{\text { объем правого яичка -объем левого яичка }}{\text { объем правого яичка }} \times 100 \%$

Существует множество публикаций, в которых пороговые значения диспропорции между тестикулами колеблются в интервале 9-20\%. Thomas et al. [18] изучили влияние степени варикоцеле на выраженность гипотрофии яичка. Авторы пришли к выводу, что задержка роста тестикул развивается у каждого четвертого пациента с варикоцеле вне зависимости от степени варикоцеле. Исследования динамики развития уменьшения объема яичка позволило авторам рекомендовать формировать показания к варикоцелэктомии при достижении индекса атрофии уровня $15 \%$ и более. Alukal et al. [19] опубликовали результаты исследования, которые демонстрируют отсутствие взаимосвязи между степенью выраженности тестикулярной гипотрофии и степенью варикоцеле. В редакционном комментарии указывается на недостаточность критериев отбора основанных на изучении объема и необходимость прямого исследования тестикулярной функции у больных с варикоцеле для определения показаний к варикоцелэктомии.

Через два года Diamond et al. [20] coобщают о результатах исследования параметров спермы у подростков с варикоцеле и различной степенью уменьшения объема яичка. Авторы делают вывод о том, что диспропорция тестикулярного объема 10\% и более высоковероятно сопровождается патоспермией. Коллеги предлагают считать индекс тестикулярной атрофии 10\% границей, за которой продолжение наблюдения нецелесообразно и необходимо использовать активную хирургическую тактику.

В 2009 году Коган М.И. с соавторами опубликовали серию статей [21-23] посвященных исследованию очень похожего по дизайну с исследованием Diamond. Результатами исследования явилось предложение считать пороговым значением индекс атрофии $>8 \%$, поскольку по данным авторов во всех случаях, когда индекс атрофии достигал значения $>8 \%$, у подростков с варикоцеле выявлялись патологические отклонения в спермограмме.

Практически одновременно с работами, посвященными значению тестикулярной асимметрии для определения показаний к хирургическому лечению, 
появляется ряд публикаций, в которых авторы демонстрируют возможность исчезновения или уменьшения асимметрии тестикулярного объема в процессе роста подростка. Существуют исследования, авторы которых считают возможным наблюдать за пациентами с индексом атрофии более $20 \%$ в течение трех лет, так как у 71\% наблюдавшихся подростков отмечалось сокращение разницы объемов яичек в процессе наблюдения [24].

Возможность консервативного ведения пациентов с наличием уменышения объема яичка на стороне варикоцеле высказали Preston et al. [25] опираясь на данные полученные ими в ретроспективном обзоре. Авторы выявили высокую вероятность исчезновения асимметрии роста в процессе динамического наблюдения, что позволило им предположить, что однократного ультразвукового исследования не достаточно для формирования показаний к варикоцелэктомии.

Другие исследования подтверждают возможность спонтанного сокращения диспропорции, но сообщают о существенно более низкой частоте явления [26], при этом отмечается возможность нарастания атрофии в процессе динамического наблюдения у 35\% пациентов.

Van Batavia et al. [27] изучили влияние стадии пубертатного периода по Таннеру на выраженность и вероятность выявления асимметрии тестикулярного объема. Результаты исследова- ния демонстрируют, что у детей находящихся в стадии I-III по Таннеру вероятность выявления уменьшения объема яичка выше, чем у пациентов находящихся в стадии IV-V. У подростков имеющих стадию IV-V по Таннеру вероятность уменьшения степени выраженности асимметрии объема яичек в процессе динамического наблюдения выше, чем у мальчиков со стадией пубертата I-III.

Таким образом, уменьшение объема яичка на стороне варикоцеле является важным критерием отбора больных, нуждающихся в варикоцелэктомии, однако накопленные сегодня научные данные не позволяют использовать критерий как единственный. Основными причинами недостаточной достоверности уменьшения тестикулярного объема у детей и подростков является возможность спонтанного восстановления объема в процессе роста и отсутствие общепринятого значения индекса тестикулярной атрофии. Накопленный опыт и изначальное понимание исследователями недостаточной специфичности уменьшения объема яичка - как критерия повреждения тестикулярной ткани у подростков явились причиной поисков альтернативных тестов, которые могли бы отражать выраженность тестикулярной альтерации при варикоцеле.

В середине 90-х годов внимание детских урологов привлекло изучение параметров кровотока яичка с помощью 
ультразвукового исследования, с целью изучения их влияния на параметры спермограмм. Paduch et al. [28] представили результаты исследования спермы у 74 подростков в возрасте от17 до 19 лет, которые были разделены на две группы - группа контроля (здоровые подростки) и юноши с варикоцеле. Авторы выявили отрицательную обратную связь между параметрами спермограммы и значением максимальной скорости ретроградного кровотока (МСРК). Однако, кроме констатации факта наличия указанной связи авторы не привели количественных показателей МСРК, которые могли бы быть использованы как маркеры отбора пациентов для варикоцелэктомии.

Только через 10 лет Kozakowski et al. [29] исследовали совокупное влияние МСРК и индекса атрофии яичка на прогноз дальнейшего развития тестикул. Авторы продемонстрировали, что при наличии МСРК выше 38 см/с и индекса атрофии 20\% нет вероятности при продолжении динамического наблюдения уменьшения индекса атрофии. Эти результаты позволили авторам предложить выполнение варикоцелэктомии подросткам при выявлении по данным ультразвукового исследования индекса атрофии 20\% и более и МСРК выше $38 \mathrm{~cm} / \mathrm{c}$.

B 2013 г. Van Batavia et al. представили результаты наблюдения за подростками, которым исследовали влияние различных значений индекса атрофии на фоне МСРК 38 см/с и выше на пер- спективы спонтанного исчезновения асимметрии объемов яичек. Авторы пришли к выводу, что уже при наличии индекса атрофии 15\% и МСРК 38 cм/c и выше продолжение динамического наблюдения нецелесообразно [30]. Авторы в следующей своей публикации изучили влияние МСРК, определяемого в послеоперационном периоде, на вероятность и выраженность рецидива варикоцеле и тестикулярной асимметрии. Van Batavia et al. [31] установили, что при выявлении после варикоцелэктомии МСРК выше $20 \mathrm{~cm} / \mathrm{c}$ высоковероятно будет определять сохранение асимметрии объема яичек и развитие рецидива.

Анализ литературы, посвященной проблеме варикоцеле у детей и подростков демонстрирует завидное постоянство задач, которые ставили и ставят перед собой исследователи сегодня и пол-века назад. Практически все исследователи начинают с фраз посвященных отсутствию достоверных данных о прямом негативном воздействии варикоцеле на репродуктивную функцию, но высказывают уверенность в необходимости в части случаев выполнять с профилактической целью варикоцелэктомию у детей и подростков. Это и определяет актуальность с их точки зрения поиск маркеров, которые бы можно было бы использовать для отбора подростков, нуждающихся в операции в условиях, когда существенно ограничено прямое исследование состояния репродуктивной функции. 
Другой важной особенностью подросткового варикоцеле является существенный разрыв между сроками хирургии и временем, когда возможно будет оценить окончательный результат в части сохранения фертильности.

Описанные проблемы осложняются неоднородностью изучаемой группы с точки зрения полового развития подростка в момент выявления и лечения варикоцеле.

Waalkes et al. [32] провели анализ существующих современных подходов в лечении варикоцеле у детей и подростков. По их данным сегодня показания к варикоцелэктомии связаны с существованием тестикулярной гипотрофии $20 \%$ и более на стороне варикоцеле в течение года и симтоматическое варикоцеле. Сочетание МСРК 38 см/с и гипотрофии яичка равное 20\% склоняет лечебную тактику в сторону хирургического лечения. При выявлении МСРК менее $30 \mathrm{~cm} / \mathrm{c}$ и тестикулярной асимметрии менее 20\% необходимо динамическое наблюдение в течение года и только при ухудшении указанных показателей показана варикоцелэктомии. В остальных случаях целесообразно наблюдение до возраста, когда будет возможно исследование параметров спермограммы.

На фоне огромного массива накопленных данных о целесообразности использования широкого спектра иссле- дования для решения вопроса о тактике ведения пациентов с варикоцеле удивительными выглядят результаты изучения практических подходов используемых американскими детскими урологами. Coutinho et al. [33] опросили 74 практикующих детских урологов. Согласно полученным ответам только 49\% из опрошенных использовали ультразвуковое исследование органов мошонки у пациентов с варикоцеле, $38 \%$ для определения тестикулярного объема применяли орхидометр Прадера. Почти треть опрошенных предлагают варикоцелэктомию сразу после выявления тестикулярной асимметрии. 57\% детских урологов никогда не направлял своих пациентов для выполнения анализа спермы. Полученные данные демонстрируют существенный разрыв между накопленным научным потенциалом и его практическим использованием в лечении детей и подростков с варикоцеле.

Нам представляется целесообразным продолжение активного поиска оптимальных сочетаний маркеров повреждения тестикулярной ткани при определении тактики ведения пациентов в зависимости от стадии полового созревания. С другой стороны целесообразна разработка методики мониторирования динамики изменения репродуктивной функции в отдаленном периоде у подростков. 


\section{ЛИТЕРАТУРА}

1. A Doppler-based study on the prevalence of varicocele in German children and adolescents / D. Pfeiffer, J. Berger, C. Schoop et al. // Andrologia. - 2006. - Vol.38. - P.13.

2. Tekgül S, Riedmiller E, Gerharz P, et al. In: Guidelines on Paediatric Urology. Arnhem, The Netherlands: European Association of Urology, European Society for Paediatric Urology // 2009. - P.23.

3. World Health Organization. The influence of varicocele on parameters of fertility in a large group of men presenting to infertility clinics // Fertil Steril. - 1992. Vol.57. - P.1289.

4. Sakamoto, H. Relationship between testicular volume and varicocele in patients with infertility / H. Sakamoto, Y. Ogawa, H. Yoshida // Urology. - 2008. - Vol.71 P.104.

5. The effect of varicocele repair on testicular volume in children and adolescents with varicocele / S. Cayan, E. Akbay, M. Bozlu et al. // J Urol. - 2002. Vol.168. - P.731.

6. Testis volumes, semen quality, and hormonal patterns in adolescents with and without a varicocele / L.C. Haans, J.S. Laven, W.P. Mali et al. // Fertil Steril. 1991. - Vol.56. - P.731.

7. Update on treatment of varicocele: counselling as effective as occlusion of the vena spermatica / E. Nieschlag, L. Hertle, A. Fischedick et al. // Hum Reprod. 1998. - Vol.13. - P.2147.

8. Bogaert, G. Pubertal screening and treatment for varicocele do not improve chance of paternity as adult / G. Bogaert, C. Orye, G. De Win // J Urol. - 2013, Jun. - Vol.189(6). - P.2298-2303.

9. Kolon, T.F. The adolescent varicocele - a Shakespearean tragedy or much ado about nothing? / T.F. Kolon // J Urol. - 2013,Jun. - Vol.189(6). - P.2024-2025.

10. Hösli, P.O. Varicocele - results following early treatment of children and adolescents / P.O. Hösli // Z Kinderchir. 1988,Jun. - Vol.43(3). - P.213-215.

11. Surgical repair of varicocele at puberty: preventive treatment for fertility improvement / A. Okuyama, M. Nakamura, M. Namiki et al. // J Urol. - 1988,Mar. Vol.139(3). - P.562-564.

12. Hösli, P.O. Early treatment of varicocele in children and adolescents / P.O. Hösli // Helv Chir Acta. - 1989,Jun. Vol.56(1-2). - P.229-233.

13. Мазо, Е. Б. Роль нарушений минералоглюкокортикоидной функции надпочечников в развитии бесплодия у больных с левосторонним варикоцеле / Е.Б. Мазо, М.В. Корякин, Ю.В. Кудрявцев // Урология и нефрология. 1989. - №2 . - C.38-45.

14. Kass, E.J. Reversal of testicular growth failure by varicocele ligation / E.J. Kass, A.B.Belman // J Urol. 1987,Mar. - Vol.137(3). - P.475-476.

15. Sigman, M. Ipsilateral testicular hypotrophy is associated with decreased sperm counts in infertile men with varicoceles / M. Sigman, J.P. Jarow // J Urol. 1997,Aug. - Vol.158(2). - P.605-607. 
16. Kass, E.J. Adolescent varicocele: objective indications for treatment / E.J. Kass, J.E. Freitas, J.B. Bour // J Urol. - 1989. - Vol.142. - P.579-582.

17. Costabile, R.A. Testicular volume assessment in the adolescent with a varicocele / R.A. Costabile, S. Skoog, M. Radowich // J Urol. - 1992, May. - Vol.147 (5). - P.1348-1350.

18. Thomas, J.C. Testicular growth arrest and adolescent varicocele: does varicocele size make a difference? / J.C. Thomas, J.S. Elder // J Urol. - 2002. Vol.168. - P.1689-1691.

19. Testicular hypotrophy does not correlate with grade of adolescent varicocele / J.P. Alukal, D. Zurakowski, A. Atala et al. // J Urol. - 2005,Dec. - Vol.174(6). P.2367-2370.

20. Relationship of varicocele grade and testicular hypotrophy to semen parameters in adolescents / D.A. Diamond, D. Zurakowski, S.B. Bauer et al. // J Urol. - 2007. - Vol.178. - P.1584-1588.

21. Results of 104 adolescents with varicocele randomised to surgery or observation and followed up for 18 months / M.I. Kogan, A. Afoko, D.V. Siziakin et al. // 24st Annual EUA Congress, Stockholm Sweden. - 2009. - Abstract 100 Eur Urol Suppl, 2009. - P.145.

22. Adolescent Varicocele: When should we intervene surgically? / M.I. Kogan, A. Afoko, D.V. Siziakin et al. // 24st Annual EUA Congress, Stockholm Sweden. 2009. - Abstract 100 Eur Urol Suppl, 2009. - P.98.
23. Варикоцеле: противоречия и проблемы / М.И. Коган, А.А. Алвин, А. Афоко и др. // Урология. - 2009. - №6. - С.67-72.

24. 3rd Transient asynchronous testicular growth in adolescent males with a varicocele / T.F. Kolon, M.R. Clement, L. Cartwrightet al. // J Urol. - 2008. - Vol.180. - P.1111-1114.

25. Conservative management of adolescent varicoceles: a retrospective review / M.A. Preston, T. Carnat, T. Flood et al. // Urology. - 2008,Jul. - Vol.72(1). - P.77-80.

26. Testicular asymmetry and adolescent varicoceles managed expectantly / S.A. Poon, C.K. Gjertson, M.A.Mercado et al. // J Urol. - 2010. - Vol.183. - P.731734.

27. Adolescent varicocele: influence of Tanner stage at presentation on the presence, development, worsening and/or improvement of testicular hypotrophy without surgical intervention / J.P. Van Batavia, S.L. Woldu, P.M. Raimondi et al. // J Urol. - 2010,Oct. - Vol.184(4 Suppl). P.1727-1732.

28. Paduch, D.A. Semen analysis in young men with varicocele: preliminary study / D.A. Paduch, J. Niedzielski // J Urol. - 1996,Aug. - Vol.156 (2 Pt 2). P.788-790.

29. Peak retrograde flow: a novel predictor of persistent, progressive and new onset asymmetry in adolescent varicocele / K.A. Kozakowski, C.K. Gjertson, G.J, Decastro et al. // J Urol. - 2009,Jun. Vol.181(6). - P.2717-2722.

30. Adolescent varicocele - is the 20/38 harbinger a durable predictor of 
testicular asymmetry? / J.P. Van Batavia, G. Badalato, A. Fast, K.I. Glassberg // J Urol. - 2013, May. - Vol.189(5). P.1897-1901.

31. Incidence, significance and natural history of persistent retrograde venous flow after varicocelectomy in children and adolescents: correlation with catch-up growth / J.P. Van Batavia, A.M. Fast, S.N. Nees et al. // J Urol. - 2013,Aug. Vol.190(2). - P.689-695.
32. Waalkes, R. Varicocele in adolescents: a review and guideline for the daily practice / R. Waalkes, I.F. Manea, J.M. Nijman // Arch Esp Urol. - 2012, Dec. - Vol.65(10). - P.859-871.

33. Variations in the management of asymptomatic adolescent grade 2 or 3 left varicoceles: A survey of practitioners / K. Coutinho, D. McLeod, K. Stensland, J.A. Stock // J Pediatr Urol. - 2013,Dec Vol.11 - P.203-205. 